$3^{\circ}$ Fucteur de puissance. - - Le courant est presque décalé de $90^{\circ}$ en avant de la tension, les pertes étant très faibles par rapfort à la puissance apparente; le facteur de puissance crô̂l avec la lension car les pertes croissent plus que proportionnellement à la tension.

4o Pertes. - Les pertes par conductance croissent très vite avec la tension et dépendent d'ailleurs énormément des condilions atmosphériques; le lemps élail beau et sec lors des essais.

$5^{\circ}$ Détermination d'apparilion d'effluves. - La formule connue de Peek donne:

$$
e_{0}=g_{0} \cdot m_{0} \cdot r \cdot d \cdot \log _{\mathrm{e}} \frac{s}{r}
$$

oì $e_{0}$ est la tension simple; $g_{0}$ le gradient de rupture de l'air par centimètre de circonférence du conducteur, soit $21,1 \mathrm{KV} . / \mathrm{c} / \mathrm{m}$; $m_{0}$ un facteur dépendant de l'état de la surface du conducteur; $r$ le rayon du conducteur en centimètres, $d$ le coefficient de densité de l'air, soil : $\frac{3,926 \times \mathrm{H}}{273+t}$ où $\mathrm{H}$ est la pression atmosphérique en centimètre de colonne de mercure et $t$ la température de l'air en degrés centésimaux; $s$ la distance entre conducteurs en centimètres.
On obtient ainsi pour les conditions atmosphériques des essais, 102.000 volts par phase, soit 176.000 volts entre phases. Par mauvais temps, il faut réduire cette valeur de $20 \%$ environ, ce qui donne 140.000 volts ; lors des essais ; il n'a en effet, pas été constalé d'effluves visibles, même de nuit.

Une pointe de tension a été poussẻe à 154.000 volts sans rien constater.

$6^{\circ}$ Un fait intéressant à signaler, quoique normal, est que en coupant l'excitation de l'excitatrice de l'alternateur servant aux essais, la capacité du système en service depuis ChancyPougny jusqu'à Sombernon, suffit à donner une tension de 8.600 volts aux bornes de l'alternateur par auto-excitation et 114.500 volts à Jeanne-Rose. Pour baisser la tension, on a inversé le sens de l'excitation de l'excitatrice et on a pu atteindre ainsi 5.200 volts à l'alternateur et 71.000 volts à Jeanne-Rose.

Cette ligne est en service depuis ces essais sans incident, le neutre haute tension est mis directement à la terre au départ et à l'arrivée.

Ajoutons que sa capacité de transport est de 29.000 KVA. pour un facteur de puissance à l'arrivée de 0,90 avec une chute de tension de $5 \%$ et un rendement de $0,95 \%$.

\title{
Aménagement du Rhin de Bâle à Strasbourg et à Lauterbourg au moyen d'une Méthode nouvelle de canalisation des Fleuves
}

I la suile de la publication dans La Houille Blanche, de l'éfude de II. MAul sur l'Aménagement du Rhin, nous recevons de la Société Regionale d'Eiudes du Rhin, une lettre que nous publions ci-après in.extenso.

Nancy, le 16 septembre 1926 .

Monsieur le Rédacteur en chef,

Votre revue a publié dans ses numéros $109-110$ el suivants, sous la signature de M. Mähl, ingénieur, un projet d'aménagement du Rhin de Bâle à Strasbourg et à Lauterbourg, contenant des affirmations, tout au moins inexacles, sur le projet du grand canal d'Alsace.

En raison de l'autorité de votre publication, j'ai l'honneur de vous donner, ci-dessous, notre réponse aux critiques de M. Mähl, et je me permets de compter sur votre haut esprit d'équité et d'impartialité pour l'insérer dans le prochain numéro de la Houille Blanche à la place où ont paru les articles de M. Mähl.

La Société Régionale d'Etudes du Rhin, constituée en 1922, a Nancy, par l'ensemble des intéressés pour l'étude et la mise à exéculion immédiate du meilleur mode d'aménagement du fleuve, ne veut pas ouvrir aujourd'hui avec M. Mähl une polémique qui n'a pas de raison d'être, la cause êtant depuịs longtemps entendue et la discussion close.
Elle se borne à remarquer que le projet du grand canal d'Alsace si âprement critiqué par M. Mähl, n'a été adopté par elle qu'après de longues recherches et études comparées, et sur l'avis parfaitement unanime et désintéressé tant de ses propres conseillers techniques que des plus hautes compétences françaises en matière de travaux publics ; les divers plans d'aménagement du fleuve ont notamment été examinés à fond et comparés par le Conseil supérieur des Travaux publics qui a définitivement adopté le projet du canal d'Alsace. Or, ce Conseil comprend, à côté des membres les plus qualifiés et les plus éminents de l'Administration des Travaux publics, des parlementaires, des Conseillers d'Etat, des représentants qualifiés de tous nos grands intérêts régionaux :

Présidents de Chambres de commerce, Directeurs de Compagnies de chemins de fer, de Sociétés de transports et de navigation, Entrepreneurs, Electriciens, Inspecteurs des finances.....

Depuis, le projet a été soumis et examiné dans tous ses détails par la Commission centrale du Rhin, organisme international qui a charge de veiller sur tous les grands intérèts internationaux auxquels peut toucher le fleuve. Cet organisme comprend autant d'Allemands que de Français, il comprend en outre des Suisses, des Belges, des Hollandais et encore des Anglais et des Italiens; c'est dire qu'il présente toute garantie d'indépendance et que ses décisions offrent toute sécurité pour conserver au Rhin son caractère international qui est maintenant mieux défini et plus jalousement gardé qu'il ne l'a jamais été. Or, la Commission centrale 
a donné son adhésion entière au projet du grand caural d'Mlsace dans sa séance du 29 avril 1925.

C'est pourquoi nous répétons que, malgré les efforls lardifs de $M$ Mähl, la discussion est aujourd'hui définitivement close.

Il nous serait facile de reprendre point par point le projet présenté par M. Mähl et de montrer qu'il ne repose sur aucune base sérieuse mais au contraire sur une série d'hypothèses non vérifiées. Ce projet contient d'ailleurs des lacunes et des erreurs si manifestes qu'elles ne résisteraient pas à un examen approfondi. Encore une fois, nous ne le voulons pas.

Entre les deux thèses soutenues par M. Mähl et par l'unanimité des compétences dont nous parlons ci-dessus, le lecteur n'aura aucune peine à discerner de quel côté se trouve la vérité.

Mais les affirmations lancées par M. Mähl dans les arlicles publiés nous paraissent tellement audacieuses que nous croyons devoir retorquer, ci-dessous, quelques-unes des déclarations les plus inexactes, qui pourraient trouver créance dans l'esprit des lecteurs non avertis.

$1^{0}$ M. Mähl qualifie le grand canal d'Alsace " d'œuvre antisociale et pouvant avoir les conséquences les plus épouvantables pour la tranquillité universelle dans l'avenir ". Il affecte de croire que la navigation n'y sera pas internationale comme sur e reste du fleuve et que le projet serait certainement condamné il était soumis à l'examen critique de la Société des Nations.

C'est vraiment prendre le contrepied exact de la réalité : le projet, nous l'avons rappelé, a été examiné de très près par la Commission centrale du Rhin, qui, dans le cas particulier, est l'organisme international tout spécialement compétent et, d'ailleurs, créé tout exprès pour l'examen de ces questions puisque :

a) Il comprend tous les riverains du Rhin, avec des effectifs proportionnés à leurs intérêts.

b) Il réunit toutes les personnalités connaissant à fond ces questions très spéciales. L'examen souhaité par $M$. Mähl a donc déjà eu lieu et il a abouti à l'approbation pure et simple du projet du canal d'Alsace;

$2^{\circ}$ Toujours d'après M. Mähl, le même projet serait " une hérésie économique ": ne permettant de récupérer que $48 \%$ de la puissance brute totale du fleuve, il en perdrait irrémédiablement et pour le seul plaisir $52 \%$, plus de la moitié.

Les chiffres de M. Mähl sont inexacts : le débit normal absorhé par le grand canal d'Alsace n'est pas plus de 815 mètres cubes que la chute disponible de 90 mètres.

Nous renvoyons sur ces points - comme sur tous ceux que nous pourrons avoir à poser en contradiction avec M. Mähl au projet d'exécution très détaillé du Grand Canal d'Alsace, projet dressé par un organisme aussi indépendant que qualifié el dont les bases certaines n'ont pu être l'objet du moindre doule au cours des nombreux examens, tant nationaux qu'inlernationaux - et en particulier allemands — qu'il a eu à subir.

En réalité - sans faire état comme M. Mähl de la régularisation future du lac de Constance - le Grand Canal d'Alsace récupère plus des $2 / 3$ de l'énergie brute totale disponible.

Résultat remarquable et qui représente le maximum de ce que l'on peut faire économiquement.
M. Mähl prétend, il est vrai, le contraire, mais il part de données lausses, se basant notamment sur un débit de plus de $1.500 \mathrm{~m}^{3}$ pendant 4 mois par an, alors que ce débil existe à peine pendant 2 mois el le moindre examen un peu poussé, la première élude séricuse de ses suggestions - qui reviennent en somme à aménager un gigantesque canal pour y faire passer tout le fleuve avec ses plus fortes crues et non pas seulement un débit constant et strictement limité comme dans le cas du Canal d'Alsace - montre immédiatement de quel côté se trouve la véritable "hérésie économique".

$3^{\circ}$ Toujours d’après MI. Mähl, la séçurité des riverains du Canal d'Alsace serait des plus précaires et les changements apportés par ce travail à l'état hydrologique naturel de la plaine du Rhin auraient les plus graves effets pour les habitants, et notamment les habitants de la rive droite. Le projet Mähl offrirait par contre sécurité, sauvegarderait les droits des riverains et leur créerait mème des avantages incomparables.

Là encore, il faut prendre cxactement l'opposé des affirmations de M. Mähl si l’on veut retrouver la réalité.

Sur Lous ces points, le projet Mähl est bien inférieur à celui dı Grand Canal.

a) Par rapport au terrain naturel, les retenues projetées par M. Mähl sont bien plus fortes que celles du Grand Canal.

Dans l'exemple purement schématique qu'il donne de l'aménagement d'un bief de 10 kilos avec une pente moyenne de $0 \mathrm{~m} .76$ par kilo, c'est-à-dire d'une chute brute disponible de $7 \mathrm{~m} .80$ (p. 12, No janvier), M. Mähl admet au droit de l'usine une revanche maximum au-dessus du T. N. de $7 \mathrm{~m} .80$ égale à la hauteur de la chute. Par ailleurs il fixe plusieurs des chutes à aménager à $12 \mathrm{~m}$. Donc, dans son projet, la retenue des eaux au droit de ces usines atteindrait $12 \mathrm{~m}$. par rapport au T. N. alors que, pour le Grand Canal, la mème revanche maximum a été systématiquement limitée à $7 \mathrm{~m}$. La revanche que $M$. Mähl estime dangereuse pour le Grand Canal n'est guère que la moitié de celle qu'il considère comme inoffensive pour son propre projet.

Encore ne faut-il pas oublier que, dans le Grand Canal, le débit et par suite le courant seront strictement limités, tandis que le fleuve endigué de M. Mähl aurait à écouler entre ses digues menacées le flot des plus grandes crues : avec une crue de $5.000 \mathrm{~m}^{3}$ seconde et la section mouillée inférieure à $2.000 \mathrm{~m}^{3}$ réalisée à l'aval de chaque barrage, la vitesse du courant atteindrait $2 \mathrm{~m} .50$ à la seconde (contre $1 \mathrm{~m}$. 20 pour le canal), c'està-dire qu'elle corroderait infailliblement les digues et conduirait vile à une rupture d'autant plus redoutable que la revanche sur le T. N. serait plus grande et le cube emmagasiné plus important: donc, dans le cas du Grand Canal d'Alsace, revanche maximum de $7 \mathrm{~m}$., vitesse maximum de $1 \mathrm{~m}$. 20, cube d'eau maximum par bief de $9.000 .000 \mathrm{~m}^{3}$; dans le cas du projet Mähl revanche pouvant atteindre $12 \mathrm{~m}$. vilesse maximum dépassant $2 \mathrm{~m}$. 50, cube d'eau par bief dépassant $30.000 .000 \mathrm{~m}^{3}$. Nous laissons au lecteur le soin de conclure où est le véritable danger.

b) A l'amont de chaque barrage, dans le projet Mähl, le plan d'eau serait relevé au moment des pointes, c'est-à-dire chaque jour, de toute la hauteur du barrage, soit de la hauteur de la chute admise, augmentée de la revanche des berges sur le plan d'eau, c'est-à-dire d'une hauteur pouvant alteindre $16 \mathrm{~m}$. (12 m. de chute plus $4 \mathrm{~m}$. de revanche).

A l'aval même des barrages, le plan d'eau serait relevé de $10 \mathrm{~m}$. par rapport au plan d'eau étiage actuel. 
Le projet du Grand Canal ne prévoit rien de semblable et c'est lui que l'on vient suspecter de pouvoir nuire aux riverains du fleuve.

Que penseront ces derniers d'un projet qui les menace directement d'une formidable surélévation du plan d'eau du fleuve, variant suivant les points de 10 à $16 \mathrm{~m}$. ?

L'auteur du projet a d'ailleurs entrevu ces dangers, et, pour y parer, il propose d'enloncer dans les digues d'exhaussement un rideau de palpanches métalliques de $5 \mathrm{~m}$. de hauteur, moyen déjà beaucoup plus coûteux que l'auteur ne l'a estimé, mais qui, pour ètre efficace, devrait envisager des hauteurs de rideaux considérables et par suite complètement impraticables.

$4^{0}$ Est-il besoin d'ajouter que les chiffres avancés par M. Màhl tant pour établir le prix de revient probable du Grand Canal d'Alsace que pour justifier son propre projet sont purement fantaisistes et ne sauraient supporter un examen sérieux.

En ce qui concerne l'évaluation des dépenses du Grand Canal, nous renvoyons encore le lecteur au projet complet, établi avec toutes les précisions voulues, dont l'étude économique, très serrée, permet d'espérer une mise en œuvre progressive et sûre dont l'équilibre financier ne laissera rien à désierr.

Cet équilibre financier a justement été et constitue toujours l'objet des études les plus suivies et les plus attentives de la Société d'Etudes et ce ne sont pas les insinuations injurieuses de M. Mähl qui y changeront quelque chose : quoi qu'en pense celui-ci, la Société d'Etudes n'a rien à voir avec les entreprises plus ou moins douteuses, et qui, sachant d'avance le projet non viable, n'hésiteraient pourtant pas à l'engager pour tirer leur profit des travaux et des tantièmes à toucher en tout état de cause. La constitution de la Société d'Etudes est le meilleur garant que les travaux ne seront engagés qu'à bon escient et sur les bases économiques les plus solides.

Il nous semble que M. Mähl, plutôt que de se lancer dans des critiques aussi acerbes contre la Société d'Etudes aurait mieux fait d'essayer une évaluation raisonnable et la moins inexacte possible de son propre projet, et notamment de ses 11 barrages en travers du Rhin, dont la plupart auraient, d'après ses prévisions, $22 \mathrm{~m}$. de hauteur utile pour $200 \mathrm{~m}$. de longueur et reposeraient sur un sol formé de gros cailloux du Rhin, c'est-à-dire essentiellement impropre à supporter avec sécurité de tels ouvrages (ne pas oublier que si le barrage de Kembs est facile à construire c'est parce qu'à cet endroit l'argile géologique affleure presque le fond de la rivière, cette couche plonge très rapidement à l'aval, et que des sondages de plus de $50 \mathrm{~m}$. de profondeur n'ont pas permis de la retrouver à hauteur de Colmar, ni de Strasbourg (1).

(1) La simple vue des figures 13 et 14 de l'article de M. Mähl (Houille Blanche, $\mathrm{n}^{\circ}$ de mai-juin, p. 80), met en évidence l'idée foncièrement inexacte qu'il se fait de la géologie de la plaine d'Alsace; sur ces figures "l'argile compacte du substratum " est figurée très près du fond du fleuve, alors que cette disposition exceptionnellement favorable ne se rencontre en réalité que tout à fait à l'amont, dans la région de Bâle. $5^{\circ}$ M. Mähl accuse encore le projet du Grand Canal d'être dangereux pour les riverains de la rive droite en cas d'inondation.

Cette accusation est, comme les autres, dénuée de tout fondement : le tracé du Grand Canal respecte presque en entier le champ d'inondation actuel du fleuve : et dans les quelques points où il n'en est pas ainsi, on s'est assuré en établissant le projet que les empiètements faits ne pouvaient avoir aucune influence sur le cours des eaux. Les meilleurs juges en cette matière ne sont-ils pas les représentants des intéressés, c'est-à-dire la Commission Centrale du Rhin, qui a déjà tranché en écartant tous ces périls imaginaires.

$6^{\circ}$ Enfin, la Société d'Etudes proteste de la façon la plus formelle contre les affirmations de M. Mähl qui la représente comme n'ayant aucun intérêt - au contraire - à voir réalisée la voie navigable entre Strasbourg et la Suisse, et se désintéressant de ce qui se fera à l'amont de Bâle, notamment au lac de Constance.

Le seul sous-titre de la Société "Navigation-Force motriceIrrigation " comme sa composition, suffisent à répondre à la première critique. La Société comprend les représentants de la Navigation française sur le Rhin : ajouterons-nous, ce qui est l'évidence même, que le Grand Canal sera la voie incomparable qui permettra bientôt à la grande navigation rhénane de remonter en toute facilité jusqu'à Bâle.

Quant à l'aménagement du lac de Constance, la Société d'Etudes y est au premier chef intéressé puisque seul cet aménagement lui permettra de faire fonctionner à plein ses installations en hiver, pendant la période des basses eaux. De ce côté encore, l'avenir est complètement ménagé par le projet du Grand Canal d'Alsace.

Les démentis et la discussion pourraient se prolonger tout au long des affirmations de M. Mähl dans les trois articles parus dans la Houille Blanche. Mais il n'est ni dans notre rôle ni dans nos intentions d'entreprendre une réfutation au demeurant si facile. Nous avons seulement voulu, dans les quelques lignes ci-dessus, faire définitivement justice des attaques passionnées lancées par celui-ci contre un projet qui n'a que le défaut d'être essentiellement français et d'avoir recueilli l'adhésion successive des nombreux organismes nationaux et internationaux qui l'ont tous longuement examiné.

Il eût, en effet, été profondément regrettable que des affirmations aussi téméraires qu'erronées puissent troubler les esprits au moment où le projet du Grand Canal d'Alsace, enfin sorti de la période préparatoire, va entrer dans la voie féconde et active des réalisations.

Veuillez agréer, Monsieur le Rédacteur en chef, l'expression de mes sentiments les plus distingués.

Le président du Conseil d'Administration,

A. Dreux. 